\title{
Developments in the treatment of carcinoid syndrome - impact of telotristat
}

This article was published in the following Dove Press journal:

Therapeutics and Clinical Risk Management

\section{David L Chan \\ Simron Singh}

Odette Cancer Centre, Sunnybrook Health Sciences Centre, Toronto, ON, Canada
Correspondence: Simron Singh Odette Cancer Centre, Sunnybrook Health Sciences Centre, 2075 Bayview Road, Toronto, ON M4N3M5, Canada Tel + I 4164804928

Fax +I 4164806002

Email simron.singh@sunnybrook.ca

\begin{abstract}
Carcinoid syndrome occurs in $20 \%$ of patients with neuroendocrine tumors, and serotonin is usually the main causative hormonal peptide. Carcinoid syndrome, and particularly diarrhea, can significantly impact patients' quality of life. Somatostatin analogs (SSAs) are the mainstay of treatment, but are unable to ameliorate symptoms in all patients due to dose-limiting side effects and tachyphylaxis. Telotristat is a novel oral inhibitor of tryptophan hydroxylase, which is the rate-limiting enzyme in serotonin synthesis. A Phase III placebo-controlled trial of telotristat etiprate (orally at $250 \mathrm{mg}$ three times a day) showed a significant decrease in the frequency of bowel motions in treated patients with diarrhea from carcinoid syndrome. The main side effects were gastrointestinal symptoms, deranged liver function tests and depression. Treatment with $500 \mathrm{mg}$ three times a day also decreased stool frequency, but was associated with more nausea and mood disturbances. Telotristat, therefore, represents a valuable option in the management of carcinoid syndrome diarrhea refractory to SSAs, and the US Food and Drugs administration approved its use for this indication in March 2017. However, its role in somatostatin-naïve patients and in the treatment of other carcinoid syndrome symptoms (flushing and abdominal pain) remains unknown. Further research should focus on these issues as well as the safety of continuing telotristat in the context of other systemic antineoplastic therapies.
\end{abstract}

Keywords: neuroendocrine, carcinoid, telotristat, diarrhea

\section{Introduction}

Neuroendocrine tumors (NETs) are a group of heterogeneous tumors derived from enterochromaffin cells, most commonly located in the small bowel, pancreas and lung. Their incidence is $\sim 6 / 100,000 /$ year, ${ }^{1}$ with the most common sites being the small intestine, pancreas, lungs and rectum. ${ }^{1}$

Approximately $20 \%$ of patients with NETs present with carcinoid syndrome which often manifests as diarrhea, flushing, bronchospasm and abdominal pain. ${ }^{2}$ These symptoms are due to the excessive secretion of hormonal peptides by neuroendocrine cells, most commonly 5-hydroxytryptophan (serotonin). Serotonin acts as a paracrine messenger in the gut, being involved in peristaltic and secretory reflexes. ${ }^{3}$ It is the main hormone implicated in the carcinoid syndrome, although other substances such as histamine and substance P may also be involved. Patients with carcinoid syndrome usually have elevated assays of urinary 5-hydroxyindolacetic acid (5-HIAA) - a metabolite of serotonin. Over several years, serotonin can also cause fibrosis in the small bowel and heart valves - leading to bowel obstruction and carcinoid heart disease. ${ }^{4}$ Carcinoid syndrome can be severe and restrict a patient's ability to work or even leave the house due to physical limitations (such as proximity to bathrooms) or social embarrassment. Patients with NET already have reduced quality of life (QoL), and a further decrease is observed in patients with carcinoid syndrome, particularly those with diarrhea. ${ }^{5,6}$ (c)
hereby accept the Terms. Non-commercial uses of the work are permitted without any further permission from Dove Medical Press Limited, provided the work is properly attributed. For permission for commercial use of this work, please see paragraphs 4.2 and 5 of our Terms (https://www.dovepress.com/terms.php). 
There are unfortunately limited treatment options for patients with carcinoid syndrome. Supportive care options such as antidiarrheals (loperamide and diphenoxylate) only provide modest symptom relief. Somatostatin analogs (SSAs), such as octreotide and lanreotide, may reduce hormone levels and improve symptoms in many patients, but clinical and biochemical responses are often incomplete. Tachyphylaxis via internalization and downregulation of somatostatin receptors on the surface of NET cells may also result in decreased SSA efficacy with ongoing treatment. ${ }^{7}$ Dose escalation of SSAs is often employed; however, data supporting their efficacy are lacking. In addition, dose escalation can often be limited by the potential for prolongation of the QT interval on electrocardiogram and cardiac complications. Debulking of tumor (whether surgical or radiological, such as embolization of hepatic metastases) may also decrease hormonal production, but these procedures can only be performed a finite number of times in predefined anatomical locations, and the results are often short lived. Therefore, there is a pressing clinical need for systemic therapies other than SSAs and antidiarrheal agents in the treatment of carcinoid syndrome. Because serotonin plays a central role in pathogenesis, drugs targeting the serotonin synthesis pathway have been investigated in this context.

\section{Tryptophan hydroxylase - a central step in serotonin synthesis}

Tryptophan hydroxylase is an aromatic amino acid hydroxylase and is the rate-limiting enzyme in serotonin synthesis. It has been detected in the brain and enterochromaffin cells in the gut. ${ }^{8}$ It catalyzes the conversion of L-tryptophan into serotonin. The two isoforms of tryptophan - TPH1 and TPH 2 - are differentially expressed in the gut and brain and thus carry out different functions. TPH1 is primarily located in the gut in enterochromaffin cells and mast cells and is implicated in the pathogenesis of colitis. ${ }^{9}$

TPH2, on the other hand, is exclusively expressed in the nervous system - both in the brain (where serotonin plays an important role in mood regulation) and the myenteric plexus. ${ }^{10}$ It plays an important role in maintaining myenteric neurons and intestinal transit, and TPH2 knockout mice displayed reduced activity and increased anxietylike behavior. ${ }^{11,12}$ In addition, selective serotonin reuptake inhibitors - which may increase the availability of serotonin in parts of the central nervous system (CNS) - are used in the treatment of depression. Therefore, a non-selective inhibition of tryptophan hydroxylase may have deleterious effects on both the CNS (depression) and the enteric nervous system (decreased gastrointestinal motility).

\section{Telotristat etiprate: pharmacology and mechanism of action}

Telotristat etiprate is a small-molecule oral peripheral inhibitor of TPH (Lexicon Pharmaceuticals, The Woodlands, TX, USA). The oral drug, telotristat etiprate, is metabolized to telotristat ethyl by carboxylesterases and subsequently undergoes hydrolysis to form telotristat - the most active form of the drug. ${ }^{13}$ Telotristat is predominantly excreted in the feces, and its half-life is 5 hours. It is not significantly metabolized by the cytochrome P450 enzymes. It does not cross the blood-brain barrier, thus decreasing the risk of CNS adverse events (AEs) related to TPH2 inhibition.

\section{Preclinical data}

Preclinical data supported the potential utility of telotristat in carcinoid syndrome, particularly in the treatment of diarrhea. A study of male mice with experimentally induced colitis demonstrated that administration of oral telotristat etiprate resulted in significant reductions in 5-hydroxytryptamine (5-HT) concentrations in plasma as well as the small intestine. Importantly, there was no significant change in the level of 5-HT either in neurons of the myenteric plexus, gastrointestinal transit time or colonic motility. ${ }^{14}$

A second study investigated the administration of telotristat in mice with induced colitis (either by dextran sulfate sodium or infection with Trichuris muris). Mice treated with telotristat had significantly less severe colitis (regardless of the induction method), decreased 5-HT levels in the small intestine and decreased levels of both neutrophilic infiltrates and pro-inflammatory cytokines IL-1B and IL-6. ${ }^{15}$

\section{Review of Phase II trials}

The effects of telotristat in decreasing 5-HT levels in the abovementioned murine studies led to two human Phase II studies of telotristat in the clinical setting. The first study was a dose finding/expansion study held at 11 sites which prospectively randomized 23 patients to telotristat (at doses of $250 \mathrm{mg}, 350 \mathrm{mg}$ or $500 \mathrm{mg}$ three times daily) or placebo. The study included patients with carcinoid syndrome, with at least four bowel motions per day on a stable dose of octreotide long-acting release (LAR), as well as biopsy-confirmed metastatic NET. Five of the 18 patients (28\%) treated with telotristat experienced a significant decrease in the number of bowel motions, defined as $30 \%$ decrease from the baseline frequency for at least two of the 4 weeks on trial medications, compared to none of the five patients on placebo. ${ }^{16}$ Urinary 5-HIAA was decreased by at least $50 \%$ in nine of 16 patients on telotristat with elevated 5-HIAA at baseline, compared to zero of five on placebo. 
A second multicenter study enrolled 15 patients to openlabel treatment with telotristat. Patients were again required to have at least four bowel movements per day to be eligible for inclusion, but prior treatment with SSAs was not mandatory. ${ }^{17}$ Treatment was initiated at a dose of $150 \mathrm{mg}$ thrice daily, and then escalated to doses of $250 \mathrm{mg}, 350 \mathrm{mg}$ and ultimately $500 \mathrm{mg}$ depending on tolerance. A total of $43 \%$ of patients had a decrease in bowel motion frequency of $>50 \%$, and urinary 5 -HIAA was reduced by $74 \%$ compared to baseline levels at 12 weeks. Most patients progressed to the maximal dose, with common study-related toxicities being gastrointestinal upset and liver enzyme abnormalities.

These two studies suggested the potential role of telotristat etiprate in controlling diarrhea from carcinoid syndrome and showed a concomitant decrease in urinary 5-HIAA, consistent with telotristat's mechanism of action. However, these small studies were not powered to determine whether telotristat produced meaningful, significant improvement in the carcinoid symptoms which ultimately affect the patient's QoL. Moreover, the second study enrolled some patients who had not previously received SSAs, and some patients received less than the "standard" dose of SSA in current practice (two SSA-naïve patients, one patient with octreotide LAR $20 \mathrm{mg}$ every 4 weeks, and three patients with lanreotide AG $90 \mathrm{mg}$ every 3 weeks). Therefore, a large randomized clinical trial was needed to investigate whether telotristat significantly improved carcinoid symptoms in patients with metastatic NET.

\section{Phase III trial:TELESTAR}

Two randomized Phase III trials were planned-TELESTAR ${ }^{18}$ and TELECAST ${ }^{19}$ - following on from the prior Phase II trials in order to formally investigate the clinical benefit of telotristat in carcinoid syndrome.

TELESTAR was a Phase III, randomized controlled trial which enrolled 135 patients with histologically confirmed metastatic NET with documented carcinoid syndrome and at least four bowel motions per day on average. ${ }^{18}$ Eligibility criteria included a stable dose of SSA for at least 3 months before enrollment. Randomization occurred on a 1:1:1 basis to telotristat $500 \mathrm{mg}$ thrice daily, telotristat $250 \mathrm{mg}$ thrice daily or placebo. This allocated treatment was continued for 12 weeks on a double-blind basis, with all patients then offered an open-label extension (OLE) program of telotristat at $500 \mathrm{mg}$ thrice daily for 36 further weeks. The primary end point of the trial was mean reduction in number of daily bowel motions compared to baseline. Secondary end points included reduction in urinary 5-HIAA, the frequency of flushing, severity of abdominal pain and QoL questionnaires. The baseline characteristics were comparable between the three arms, with baseline bowel motion frequency being 5.2 motions per day for placebo, 6.1 for $250 \mathrm{mg}$ and 5.8 in $500 \mathrm{mg}$ (overall range 3.5-13.0). Approximately $45 \%$ of enrolled patients were receiving SSAs above the standard dose (octreotide LAR $30 \mathrm{mg}$ or lanreotide ATG $120 \mathrm{mg}$ per 4 weeks).

The mean number of bowel motions was consistently decreased in all three arms compared to baseline. This change was -1.7 in the $250 \mathrm{mg}$ arm and -2.1 in the $500 \mathrm{mg}$ arm, both of which were significantly superior to the measurement of -0.9 in the placebo arm ( $p<0.001$ for both comparisons). Urgency to defecate (measured as the proportion of days this symptom was experienced) was significantly decreased in the $500 \mathrm{mg}$ arm $(p=0.006)$ but not in the $250 \mathrm{mg}$ arm $(p=0.35)$. Urinary 5-HIAA was decreased in both treatment arms but increased in the placebo arm; the mean change was $-40.1 \mathrm{mg} / 24$ hours for $250 \mathrm{mg}$ and $-57.7 \mathrm{mg}$ for $500 \mathrm{mg}$. A post-hoc analysis showed that $78 \%$ of patients in the $250 \mathrm{mg}$ arm and $87 \%$ of patients in the $500 \mathrm{mg}$ arm experienced $\mathrm{a} \geq 30 \%$ decrease in urinary 5-HIAA compared to baseline.

Significant flushing ( $\geq 2$ episodes per day) and abdominal pain $(\geq 3 / 10)$ end points were not significantly altered by telotristat treatment although a trend toward improvement was noted. QoL, as measured by the EORTC core quality of life questionnaire version 3.0 (QLQ-C30) diarrhea subscale, was improved significantly in the $250 \mathrm{mg}$ group compared to placebo ( $p=0.039$ ) but not in the $500 \mathrm{mg}$ group $(p=0.051$ ). The global QLQ-C30 scores and the nausea and vomiting subscales were not significantly different on treatment.

While any grade treatment-emergent AEs were commonly noted in all three arms $(250 \mathrm{mg}-82 \%, 500 \mathrm{mg}-$ $93 \%$ and placebo $-87 \%$ ), this rarely led to discontinuation of study treatment (7\% in both $250 \mathrm{mg}$ and $500 \mathrm{mg}$ arms and $13 \%$ in placebo). Nausea was increased in the $500 \mathrm{mg}$ arm, and increased gamma-glutamyl transferase (GGT) was noted in both telotristat arms. Depression and confusion were more common in the $500 \mathrm{mg}$ arm, but none of the patients required new antidepressant therapy or trial discontinuation (Table 1).

As mentioned earlier, all patients enrolled on TELESTAR were offered enrollment in the OLE program after the 12-week double-blind evaluation period. A total of 115 patients were enrolled, and $31 \%$ reported a serious $\mathrm{AE}$ during treatment, with $12 \%$ discontinuing treatment. Depression-related AEs (depression, depressed mood and decreased interest) were 
Table I Incidence of overall and selected adverse events (AEs, $>10 \%)$ in the TELESTAR trial

\begin{tabular}{llll}
\hline AE & $\begin{array}{l}\text { Placebo } \\
\mathbf{( N = 4 5 ) ,}\end{array}$ & $\begin{array}{l}\text { Telotristat } \\
\mathbf{2 5 0} \mathbf{~ m g}\end{array}$ & $\begin{array}{l}\text { Telotristat } \\
\mathbf{5 0 0} \mathbf{~ m g}\end{array}$ \\
& $\mathbf{n}(\%)$ & $\begin{array}{l}\mathbf{( N = 4 5 ) ,} \\
\mathbf{n}(\%)\end{array}$ & $\begin{array}{l}\mathbf{( N = 4 5 )} \\
\mathbf{n}(\%)\end{array}$ \\
\hline All treatment-emergent AEs & $39(87)$ & $37(82)$ & $42(93)$ \\
AE resulting in discontinuation & $6(13)$ & $3(7)$ & $3(7)$ \\
AE resulting in death & $3(7)$ & $\mathrm{I}(2)$ & $\mathrm{I}(2)$ \\
Nausea & $5(11)$ & $6(13)$ & $14(3 \mathrm{I})$ \\
Abdominal pain & $8(18)$ & $5(11)$ & $10(22)$ \\
Vomiting & $4(9)$ & $2(4)$ & $5(11)$ \\
Depression & $3(7)$ & $3(7)$ & $7(16)$ \\
\hline
\end{tabular}

Note: Adapted with permission. () (2017) American Society of Clinical Oncology. All rights reserved. Kulke MH, Hörsch D, Caplin ME, et al. J Clin Oncol. Telotristat ethyl, a tryptophan hydroxylase inhibitor for the treatment of carcinoid syndrome. $2017 ; 35(1): 14-23 . .^{18}$

noted in $15 \%$ of the population, but the incidence of depression was similar in the placebo and $250 \mathrm{mg}$ groups.

TELECAST is another Phase III randomized study investigating telotristat etiprate in a similar fashion to TELESTAR. This trial was performed because a significant number of patients with carcinoid syndrome may not have bowel motions as frequently as the TELESTAR trial population, but may still suffer from other symptoms (such as fecal urgency or flushing) that may affect their QoL. Patients enrolled in TELECAST needed to have metastatic NET and at least one carcinoid syndrome-related symptom (such as flushing) but $<4$ bowel motions per day if they were treated with SSAs. The results have been presented in abstract form, but the full results have not been published to date. ${ }^{19}$ A total of 67 patients were enrolled and randomized to telotristat $250 \mathrm{mg}$ thrice daily, $500 \mathrm{mg}$ thrice daily or placebo, with a 12-week double-blind treatment period, as per TELESTAR. The primary co-end points were safety and change in urinary 5-HIAA at 12 weeks compared to baseline. Efficacy in reducing stool frequency was defined as a decrease of at least $30 \%$ for $>50 \%$ of days in the treatment period. More than $90 \%$ of enrolled patients were on SSAs, and the mean bowel motion frequency was 2.5 (range 2.2-2.8). The mean urinary 5-HIAA was again decreased compared to placebo (250 mg: mean difference $-30 \mathrm{mg} /$ day, $-54 \%$, $p<0.001$ and $500 \mathrm{mg}:-41 \mathrm{mg},-90 \%, p<0.001)$. A total of $40 \%$ of patients experienced efficacy in reducing stool frequency. There were insufficient data on non-diarrhea symptoms, and these were not specifically reported (Dr M Pavel, personal written communication, July 6, 2017). The side effect profile was generally similar to those reported earlier in the TELECAST trial with no new AEs noted.

The TELESTAR trial established that telotristat etiprate could decrease stool frequency in patients with carcinoid syndrome through inhibition of serotonin synthesis, as supported by the reduction in urinary 5-HIAA levels. This led to the approval of telotristat (at $250 \mathrm{mg}$ three times a day) by the US Food and Drugs Administration (FDA) for the treatment of carcinoid syndrome diarrhea in patients with inadequate control on SSA therapy alone.

The Telotristat Expanded Treatment for Patients with Carcinoid Syndrome Symptoms (TELEPATH) trial may provide further information regarding the long-term safety and efficacy profile of telotristat; the trial has closed to accrual, but no results have been presented to date.

\section{Discussion}

The development of telotristat has followed well-established patterns in drug development. The implication of serotonin in manifestation of carcinoid syndrome diarrhea provided the rationale for drug development. After synthesis of the agent, promising preclinical data were noted for telotristat to decrease serotonin production. The efficacy detected in Phase II trials led to a successful, well-conducted Phase III trial (TELESTAR) and subsequently FDA registration in a much-needed clinical condition with few available therapies. The fact that improvements were noted in the placebo arm is not unexpected in a patient-reported end point, although the exact number of bowel motions, being an objective number, should in theory be less susceptible to evaluation bias by the patient. This underscores the importance of double-blind, placebo-controlled trial designs for agents which primarily improve disease symptoms. The statistical analysis in TELESTAR was robust, consisting of both nonparametric analysis with the Hodges-Lehmann estimator and mean differences compared to the placebo arm to give an indication of the magnitude of symptomatic improvement.

However, there are some potential limitations to the abovementioned trials. The patient population investigated in TELESTAR was a subset of all patients with carcinoid syndrome - those who are on a stable dose of SSAs (ie, several months after presentation and without significant AEs from SSAs), with at least four bowel movements per day. While reduced stool frequency was also noted in the TELECAST trial, the significance of this is difficult to evaluate prior to full trial publication. The novel choice of end point in the TELECAST trial also impedes comparison to other trials. While the mean number of bowel movements was indeed reduced, a reduction from 6.1 to 4.4 in the $250 \mathrm{mg}$ group (the recommended FDA dose) still leaves many NET patients with significant symptoms from diarrhea. A patient with four bowel motions a day, decreased to two on telotristat, might 
experience greater improvements in their QoL compared to one who started with 10 bowel motions daily and still had eight after telotristat. Furthermore, the QLQ-C30 global QoL scores were not significantly improved on treatment (although the diarrhea subscale was improved). It is therefore hard to quantify the impact of telotristat on decreasing disruption to the life of NET patients with carcinoid syndrome. While TELESTAR was a well-conducted trial, the choice of end point means that this trial should be used as an agent to decrease bowel frequency, but not primarily to control hormonal hypersecretion or to improve other carcinoid syndromes at present.

The choice of the end point also raises important questions regarding the selection of trial end points. There is ongoing debate regarding what magnitude of change constitutes "significant improvement" in an end point, even in welldefined, objective end points such as overall survival. ${ }^{20}$ For NETs which may be relatively indolent, preserving QoL is paramount in patients with carcinoid syndrome. Some aspects of QoL, such as stool urgency impact on a patient's ability to travel freely, may be hard to quantify. The involvement of patients and advocacy groups in deciding what a significant improvement is may be critical in the development of further agents to improve patients' QoL.

\section{The current place of telotristat in the treatment paradigm of carcinoid syndrome}

As mentioned earlier, patients with carcinoid syndrome had few viable systemic options for hormonal and symptomatic control-essentially consisting of SSAs at ever-higher doses, other therapies designed to reduce tumor burden, and supportive care. Telotristat is a novel agent with demonstrated efficacy in the setting of carcinoid syndrome diarrhea despite the use of SSAs. For patients on long-acting SSAs who still have diarrhea (4+ bowel motions per day), telotristat should be considered for the improvement of diarrhea. For patients with less diarrhea ( $<4$ motions per day) on normal dose SSAs, it is currently unknown whether initiation of telotristat in addition to normal dose SSAs or escalation of SSA dose alone (eg, administration of octreotide LAR $30 \mathrm{mg}$ fortnightly) represents the superior initial strategy in terms of tolerability, safety or cost-effectiveness. While the additional benefit of telotristat has been proven in randomized trials, the side effects of gastrointestinal upset and potential mood disturbance need to be weighed up for the individual patient. Ultimately, the treatment decision may rest on the patient's comorbidities, preferences (eg, the severity of injection site discomfort experienced by the patient from SSAs) as well as local availability and cost.

\section{Unresolved issues and future directions for research}

The benefit of telotristat has been shown in NETs with severe diarrhea despite SSAs, but its benefit in other settings remains unclear. The decrease in urinary 5-HIAA may correlate with improvement in serotonin-mediated symptoms of carcinoid syndrome such as intestinal fibrosis (reduction in the frequency of small bowel obstruction and abdominal pain) and carcinoid heart disease, but given these symptoms manifest over years, it will be difficult to demonstrate a significant impact from telotristat treatment in the short term. The optimal place for telotristat in the treatment algorithm of carcinoid syndrome is also unclear. Future studies may look at initiation of both SSAs and telotristat on initial diagnosis of severe carcinoid syndrome to gain quicker control of symptoms and improve QoL, rather than waiting several months to see whether SSAs are effective. In addition, a comparison between dose escalation of SSAs and initiation of telotristat upon evidence of insufficient control on standard dose SSA would be interesting and help clinicians sequence these therapeutic options optimally. Patients who are intolerant to SSAs should theoretically benefit from telotristat as well; however, only small numbers of patients off SSA treatment have received telotristat thus far, and its tolerability and efficacy in this population deserve further investigation.

Finally, there are currently little data regarding the impact of telotristat on non-diarrhea symptoms (flushing and abdominal pain), as neither TELESTAR nor TELECAST was powered to evaluate this end point. Abdominal pain and nausea were each reported by less than half of the patients on TELESTAR. While this percentage is expected to be higher for TELECAST, the smaller enrollment and lack of formal analysis of these endpoints mean that it may be hard to draw firm conclusions on currently available data.

The successful development of telotristat should lead to further preclinical research for novel agents that can affect hormonal synthesis - not just targeted at the serotonin pathway but toward the synthesis of other vasoactive substances such as substance $\mathrm{P}$, histamine, catecholamines and prostaglandins, all of which may play a role in the pathogenesis of flushing. ${ }^{21}$

The development of new anti-tumor systemic therapies will also raise questions regarding their combination with telotristat. SSAs are often continued in combination 
with other systemic therapies upon disease progression in patients with carcinoid syndrome, and trials such as NETTER-1 (peptide receptor radionuclide therapy [PRRT]) and RADIANT-2 (everolimus) demonstrate the safety of such an approach. ${ }^{22,23}$ The feasibility of continued telotristat treatment in the setting of other standard systemic therapies (everolimus, sunitinib and PRRT) needs to be evaluated further. The impact of telotristat on tumor growth is also unclear as it has not been evaluated as an end point in trials to date. While there is little evidence to suggest that serotonin synthesis plays a part in tumor growth, evaluation of this end point in future trials is important in a disease where ongoing tumor growth may also cause symptoms and affect patient outcomes.

\section{Conclusion}

Telotristat is a novel serotonin synthesis inhibitor whose mechanism of action is distinct to that of SSAs. It represents a significant advance in the treatment of carcinoid syndrome diarrhea in patients who have inadequate control on longacting SSAs and should be considered for patients with $>4$ bowel motions per day on SSAs. The impact of telotristat on non-diarrhea carcinoid symptoms, its safety in combination with other systemic therapies, and its cost-effectiveness remain topics for future research.

\section{Acknowledgments}

We would like to acknowledge Marianne Pavel for provision of TELECAST data. No internal or external funding was received in support of this work.

\section{Disclosure}

DLC has received research funding from EMD Serono and honoraria from Ipsen. SS has received honoraria and travel funding from Ipsen, Pfizer and Novartis, and research funding from EMD Serono. The authors report no other conflicts of interest in this work.

\section{References}

1. Hallet J, Law CHL, Cukier M, Saskin R, Liu N, Singh S. Exploring the rising incidence of neuroendocrine tumors: a population-based analysis of epidemiology, metastatic presentation, and outcomes. Cancer. 2015;121(4): 589-597.

2. Halperin DM, Shen C, Dasari A, et al. Frequency of carcinoid syndrome at neuroendocrine tumour diagnosis: a population-based study. Lancet Oncol. 2017;18(4):525-534.

3. Gershon MD, Tack J. The serotonin signaling system: from basic understanding to drug development for functional GI disorders. Gastroenterology. 2007;132(1):397-414.
4. Grozinsky-Glasberg S, Grossman AB, Gross DJ. Carcinoid heart disease: from pathophysiology to treatment - 'something in the way it moves'. Neuroendocrinology. 2015;101(4):263-273.

5. Singh S, Granberg D, Wolin E, et al. Patient-reported burden of a neuroendocrine tumor (NET) diagnosis: results from the first global survey of patients with NETs. J Glob Oncol. 2016;3(1):43-53.

6. Beaumont JL, Cella D, Phan AT, Choi S, Liu Z, Yao JC. Comparison of health-related quality of life in patients with neuroendocrine tumors with quality of life in the general US population. Pancreas. 2012; 41(3):461-466.

7. Hofland LJ, Lamberts SWJ. The pathophysiological consequences of somatostatin receptor internalization and resistance. Endocr Rev. 2003; 24(1):28-47.

8. Fitzpatrick PF. Tetrahydropterin-dependent amino acid hydroxylases. Annu Rev Biochem. 1999;68:355-381.

9. Ghia J-E, Li N, Wang H, et al. Serotonin has a key role in pathogenesis of experimental colitis. Gastroenterology. 2009;137(5):1649-1660.

10. Walther DJ, Peter J-U, Bashammakh S, et al. Synthesis of serotonin by a second tryptophan hydroxylase isoform. Science. 2003; 299(5603):76.

11. Li Z, Chalazonitis A, Huang Y-Y, et al. Essential roles of enteric neuronal serotonin in gastrointestinal motility and the development/survival of enteric dopaminergic neurons. J Neurosci. 2011;31(24):8998-9009.

12. Savelieva KV, Zhao S, Pogorelov VM, et al. Genetic disruption of both tryptophan hydroxylase genes dramatically reduces serotonin and affects behavior in models sensitive to antidepressants. PLoS One. 2008;3(10): 33301.

13. Telotristat ethyl [prescribing information]. Available from: https://www. xermelo.com/Media/Default/pdfs/Product_Info_telotristat_ethyl.pdf. Accessed October 16, 2017.

14. Margolis KG, Stevanovic K, Li Z, et al. Pharmacological reduction of mucosal but not neuronal serotonin opposes inflammation in mouse intestine. Gut. 2014;63(6):928-937.

15. Kim JJ, Wang H, Terc JD, Zambrowicz B, Yang QM, Khan WI. Blocking peripheral serotonin synthesis by telotristat etiprate (LX1032/ LX1606) reduces severity of both chemical- and infection-induced intestinal inflammation. Am J Physiol Gastrointest Liver Physiol. 2015; 309(6):G455-G465.

16. Kulke MH, O'Dorisio T, Phan A, et al. Telotristat etiprate, a novel serotonin synthesis inhibitor, in patients with carcinoid syndrome and diarrhea not adequately controlled by octreotide. Endocr Relat Cancer. 2014; 21(5):705-714.

17. Pavel M, Hörsch D, Caplin M, et al. Telotristat etiprate for carcinoid syndrome: a single-arm, multicenter trial. J Clin Endocrinol Metab. 2015; 100(4):1511-1519.

18. Kulke MH, Hörsch D, Caplin ME, et al. Telotristat ethyl, a tryptophan hydroxylase inhibitor for the treatment of carcinoid syndrome. J Clin Oncol. 2017;35(1):14-23.

19. Pavel M, Gross D, Benavent M, et al. Efficacy and safety results of telotristat ethyl in patients with carcinoid syndrome during the double-blind treatment period of the TELECAST stage 3 clinical trial. Pancreas. 2017; 46(3):434.

20. Ellis LM, Bernstein DS, Voest EE, et al. American Society of Clinical Oncology perspective: raising the bar for clinical trials by defining clinically meaningful outcomes. J Clin Oncol. 2014;32(12):1277-1280.

21. Hannah-Shmouni F, Stratakis CA, Koch CA. Flushing in (neuro) endocrinology. Rev Endocr Metab Disord. 2016;17(3):373-380.

22. Strosberg J, El-Haddad G, Wolin E, et al. Phase 3 Trial of ${ }^{177}$ Lu-Dotatate for Midgut neuroendocrine tumors. N Engl J Med. 2017;376(2): 125-135.

23. Pavel ME, Hainsworth JD, Baudin E, et al. Everolimus plus octreotide long-acting repeatable for the treatment of advanced neuroendocrine tumours associated with carcinoid syndrome (RADIANT-2): a randomised, placebo-controlled, phase 3 study. Lancet. 2011; 378(9808):2005-2012. 
Therapeutics and Clinical Risk Management

Dovepress

\section{Publish your work in this journal}

Therapeutics and Clinical Risk Management is an international, peerreviewed journal of clinical therapeutics and risk management, focusing on concise rapid reporting of clinical studies in all therapeutic areas, outcomes, safety, and programs for the effective, safe, and sustained use of medicines. This journal is indexed on PubMed Central, CAS,

EMBase, Scopus and the Elsevier Bibliographic databases. The manuscript management system is completely online and includes a very quick and fair peer-review system, which is all easy to use. Visit http://www.dovepress.com/testimonials.php to read real quotes from published authors.

Submit your manuscript here: http://www.dovepress.com/therapeutics-and-clinical-risk-management-journal 